\title{
Avaliação das Interações da Fauna de Formigas em Diferentes Usos do Solo
}

\author{
Gustavo Correiro do Amaral ${ }^{1}$, André Barbosa Vargas² \& Fábio Souto de Almeida
}

Recebido em 11/08/2020 - Aceito em 14/12/2020

1 Universidade Federal Rural do Rio de Janeiro/UFRRJ, Programa de Pós-Graduação em Fitossanidade e Biotecnologia Aplicada, Instituto de Biologia, Secretaria do PPGFBA, BR 465, Km 07, Seropédica/RJ. Brasil. CEP: 23.897-000. <gustavo_correiro@yahoo.com.br, fbio_almeida@yahoo.com.br>.

2 Centro Universitário de Volta Redonda, Curso de Ciências Biológicas, Avenida Paulo Erlei Alves Abrantes, 1325, Três Poços, Volta Redonda/RJ. Brasil. CEP: 27.240-560. <andrebvargas@yahoo.com.br>.

RESUMO - O objetivo deste estudo foi avaliar as interações da fauna de formigas em uma floresta ombrófila densa, em um sistema agroflorestal diversificado, uma plantação de café sombreado por gliricídia e uma plantação de bananas que possuía vinhas de maracujá. Em cada um desses ambientes foram demarcados três transectos de $10 \mathrm{~m} \times 2 \mathrm{~m}$, onde as formigas foram coletadas e as interações ecológicas foram avaliadas. No total, foram observadas e coletadas 30 espécies de formigas, sendo a maior riqueza de espécies observada na agrofloresta, como, também, maior registro de interações. Já a menor riqueza e o menor registro de interações ocorreram no cafezal. Com base nos resultados do presente estudo, é possível constatar que, entre os sistemas de cultivo estudados, o sistema agroflorestal é o mais recomendado para a proteção da biodiversidade de formigas, por preservar maior número de espécies e de interações.

Palavras-chave: Formicidae; relações ecológicas; grupos funcionais; biodiversidade; conservação.

\section{Evaluation of Ant Fauna Interactions in Different Land Uses}

ABSTRACT - The objective of this study was to evaluate the interactions of ant fauna in a dense ombrophilous forest, a diversified agroforestry system, a gliricídia shaded coffee plantation and a banana plantation that had passion fruit vines. In each of these environments three $10 \mathrm{~m} \times 2 \mathrm{~m}$ transects were demarcated, where ants were collected and ecological interactions were evaluated. In total, 30 species of ants were observed and collected, the greatest species richness observed in agroforestry, as well as the greatest record of interactions. The smallest richness and the smallest record of interactions occurred in the coffee plantations. Based on the results of this study, it is possible to verify that, among the cultivation systems studied, the agroforestry system is the most recommended for the protection of ant biodiversity, as it preserves the largest number of species and interactions.

Keywords: Formicidae; ecological relations; functional groups; biodiversity; conservation.

\section{Evaluación de las Interacciones de la Fauna de Hormigas en Diferentes Usos de la Tierra}

RESUMEN - El objetivo de este estudio fue evaluar las interacciones de la fauna de hormigas en un bosque ombrofilo denso, un sistema agroforestal diversificado, una plantación de café con sombra de gliricídia y una plantación de plátano que tenía vides de maracuyá. En cada uno de estos ambientes se demarcaron tres transectos de $10 \mathrm{~m} \times 2 \mathrm{~m}$, donde se recolectaron hormigas y se evaluaron las interacciones ecológicas. En total, se observaron y recogieron 30 especies de hormigas, la mayor riqueza de especies observada en la agroforestería, así como el mayor registro de interacciones. La menor riqueza y el menor registro de interacciones se dieron en los cafetales. A partir de los resultados de este estudio, se puede ver que, entre los sistemas de cultivo estudiados, el sistema agroforestal es el más recomendable para la protección de la biodiversidad de las hormigas, ya que preserva el mayor número de especies e interacciones.

Palabras clave: Formicidae; relaciones ecológicas; grupos funcionales; biodiversidad; conservación. 


\section{Introdução}

A proteção da biodiversidade global é uma das questões mais importantes da atualidade, tendo em vista as dificuldades diante da perda de serviços ecossistêmicos advindos das elevadas taxas de extinção (Pimm et al., 2014). Os conceitos modernos, em geral, determinam que a biodiversidade é composta por diferentes níveis de complexidade da vida (Almeida \& Vargas, 2017). Mas os estudos que visam descrever a diversidade biológica de um local frequentemente abordam apenas a riqueza de espécies que existem na área, sem incluir a diversidade genética, de ecossistemas $e$ as interações ecológicas (Peres et al., 2011; Tabarelli et al., 2012; Rodrigues et al., 2016). Avaliar as interações ecológicas existentes em uma área é fundamental para a criação de estratégias que visem à manutenção do equilibrio dos ecossistemas e também para a utilização de interações que sejam úteis para as atividades humanas, incluindo aquelas importantes para a produção agrícola (Estrada, 2017).

A estrutura dos agroecossistemas influencia a composição das assembleias de formigas $e$, consequentemente, as interações ecológicas das quais fazem parte (Rodrigues et al., 2008; Silva, 2012). Em áreas de cultivo orgânico, diversas interações ecológicas podem ser mais frequentes que em cultivos convencionais, devido à não utilização de defensivos químicos agrícolas altamente prejudiciais à biota $e$ ao fato de os tratos culturais, geralmente, serem menos invasivos que os adotados em cultivos convencionais (Queiroz et al., 2006). Além disso, os cultivos orgânicos possuem, muitas vezes, maior riqueza de espécies cultivadas, e os agricultores mantêm a cobertura morta sobre o solo, o que também pode potencializar a ocorrência de interação predador/ presa, competição e mutualismo (Oliveira, 2013). Assim, a disponibilidade de nichos ecológicos tende a ser maior em cultivos orgânicos. Tais características tornam essas áreas interessantes para a realização de estudos sobre interações ecológicas envolvendo formigas. Ademais, potencializar interações ecológicas úteis para a produção agrícola é especialmente importante em áreas cultivadas em que a aplicação de defensivos agrícolas é limitada (Estrada, 2017).

As formigas são insetos que apresentam várias interações ecológicas úteis aos agroecossistemas (Queiroz et al., 2006). Embora existam espécies que cortam folhas ou outras partes de plantas cultivadas e, assim, causam a redução da produção agrícola e perdas econômicas (Forti, 2000; Cantarelli et al., 2008; Fujihara et al., 2013), a grande maioria das espécies de formigas apresentam outras funções que podem ser benéficas aos cultivos (Fillmann \& Sterling, 1983). A fauna de formigas contribui, por exemplo, para a ciclagem de nutrientes do solo e para a redução de populações de espécies praga, através da predação (Wilson, 1987; Hölldobler \& Wilson, 1990). Apesar do importante papel das formigas nas áreas cultivadas, existem poucos estudos sobre as interações ecológicas desses insetos nos agroecossistemas, principalmente em áreas sob manejo orgânico.

Deste modo, o objetivo deste estudo foi avaliar as interações ecológicas das assembleias de formigas em diferentes usos do solo, discutindo-se o papel desempenhado pela fauna de formigas em agroecossistemas.

\section{Material e Métodos}

O estudo foi conduzido no Sistema Integrado de Produção Agroecológica (SIPA), situado no município de Seropédica, estado do Rio de Janeiro, que apresenta 59ha e é destinado a atividades voltadas ao ensino, pesquisa e extensão relacionadas à agroecologia (Neves et al., 2005). Os tipos de solo mais frequentes na localidade são os planossolos e os argissolos vermelho-amarelos (Neves et al., 2005). Com média de $1.225 \mathrm{~mm}$ de chuva/ano, o maior volume de chuva é registrado no verão; o mês mais quente é fevereiro (média de $26,7^{\circ} \mathrm{C}$ ), e junho o mais frio (média de $20,7^{\circ} \mathrm{C}$ ); o clima é o Cwa (subtropical com inverno seco) (EMBRAPA, 2016).

As amostragens foram realizadas na estação chuvosa (janeiro e fevereiro de 2017) e na seca (julho e agosto de 2017) em quatro áreas distintas, incluindo um fragmento de floresta ombrófila densa secundária, com, aproximadamente, oito hectares; e outras três áreas cultivadas, mantidas sob manejo agroecológico. Um sistema agroflorestal diversificado (agrofloresta), um cafezal sombreado e um plantio de bananeiras foram as áreas cultivadas utilizadas no estudo. O sistema agroflorestal (0,25ha) (Nóbrega et al., 2006) apresentava espécies de porte herbáceo, arbustivo e arbóreo, incluindo espécies agrícolas 
e florestais, entre as quais destacam-se: bananeira (Musa pariadisiaca L.), guapuruvu (Schizolobium parahyba (Vell.) S.F. Blake), abacaxi (Ananas comosus (L.) Merr.), urucum (Bixa orelana L.) e mamoeiro (Carica papaya L.), entre outras. O plantio de café (Coffea conephora Pierre), com 0,23ha, apresentava sombreamento pela espécie arbórea Gliricidia sepium (Jacq.). A plantação de bananeiras (Musa pariadisiaca L.), com 0,2ha, apresentava vinhas de maracujá (Passiflora edulis Sims).

Em cada ambiente estudado, foram demarcados três transectos de $10 \times 2 \mathrm{~m}$, os quais foram vistoriados em horários e dias alternados por 10 vezes em cada área, por estação, no período das $7 \mathrm{~h}$ às $12 \mathrm{~h}$, totalizando 100 horas por estação, e um total de 200 horas. As vistorias nos transectos consistiram em observar o solo $e$ as plantas (até a altura de $1,8 \mathrm{~m}$ ), coletando as formigas encontradas e verificando as interações. Desta forma, todas as interações envolvendo formigas foram contabilizadas, e todos os táxons coletados foram mantidos em recipientes com álcool $70 \%$, para posterior identificação. As formigas foram identificadas com base na chave de Baccaro et al. (2015) e naquelas específicas para os gêneros amostrados. Ademais, foram realizadas comparações entre os espécimes coletados e outros previamente identificados, presentes na coleção Angelo Moreira da Costa Lima (CECL). Todos os outros táxons envolvidos nas interações, como as plantas, incluindo frutos e sementes, artrópodes e outros animais, foram identificados sempre que possível. Em cada transecto, ao lado de cada amostra, os atributos ambientais - profundidade de serapilheira, temperatura do ar e luminosidade - foram mensurados com um termo-higro anemômetroluxímetro digital (THAL-300).

Posteriormente, uma lista de espécies foi obtida, assim como a riqueza de espécies e as interações ecológicas. A riqueza de espécies foi comparada entre ambientes através da análise de covariância (ANCOVA), com as variáveis ambientais (temperatura do ar, luminosidade e a profundidade de serapilheira) como covariáveis, no sentido de avaliar se possíveis diferenças nos ambientes estariam relacionadas à riqueza de espécies de formigas $e$, consequentemente, às suas interações com outros organismos.

\section{Resultados e Discussão}

Foram observadas e coletadas 30 espécies de formigas no total, sendo a maior riqueza de espécies observada na agrofloresta, como também o maior registro de interações (Tabela 1). Já a menor riqueza e o menor registro de interações ocorreram no cafezal. Embora se saiba que, quanto maior a complexidade estrutural dos habitat, maior é a diversidade de espécies de formigas (Martins et al., 2011; Amaral et al., 2019; Assis et al., 2018), a análise não indicou variação significativa na riqueza de espécies entre ambientes (ANCOVA, $F=2,741 ; \mathrm{P}=0,15$, Figura 1).

Apesar das diferenças entre os ambientes, inclusive em relação à composição de espécies de plantas, elas não foram suficientes para provocar a distinção na riqueza de espécies de formigas entre as áreas estudadas durante as estações seca $e$ chuvosa, não havendo um valor significativo que mostre tal diferença.

Mesmo os ambientes sofrendo distúrbios regulares para a manutenção do plantio, a mirmecofauna no local se manteve a mesma.

Da mesma forma que a visível distinção da cobertura do solo dos ambientes estudados não foi suficiente para uma distinção da riqueza de espécies quanto à profundidade de serapilheira (ANCOVA, $\mathrm{F}=1,680 ; \mathrm{P}=0,25$ ), temperatura do ar (ANCOVA, $F=0,115 ; \mathrm{P}=0,75$ ) como constatado por Vargas et al. (2007) e pela a luminosidade (ANCOVA, $\mathrm{F}=0.568 ; \mathrm{P}=0,49$ ).

Durante o estudo, a mirmecofilia, com cochonilhas e pulgões, foi o tipo de interação ecológica mais frequentemente observada, seguida da predação (Tabela 2). Dentre as interações, a predação foi observada no bananal, na agrofloresta e na floresta secundária. As interações entre formigas e fitófagos foram observadas na agrofloresta e no cafezal, e a herbivoria na agrofloresta e na floresta secundária. Porém, esses registros foram mais expressivos na agrofloresta, que é um ambiente mais heterogêneo em comparação com o cafezal e o bananal. Sua cobertura do solo apresenta maior diversidade de material vegetal em processo de decomposição, tornando a serapilheira mais heterogênea. 
Tabela 1 - Espécies de formigas coletadas em áreas com diferentes usos do solo no SIPA, Seropédica/RJ.

\begin{tabular}{|c|c|c|c|c|}
\hline Espécies & Bananal & Agrofloresta & Cafezal & Floresta secundária \\
\hline Atta sexdens Linnaeus, 1758 & $\mathrm{x}$ & & $\mathrm{X}$ & $\mathrm{X}$ \\
\hline Camponotus crassus Mayr, 1862 & $\mathrm{X}$ & $\mathrm{x}$ & $\mathrm{x}$ & \\
\hline Camponotus melanoticus Emery, 1894 & $\mathrm{X}$ & & & \\
\hline Camponotus novogranadensis Mayr, 1870 & & $\mathrm{x}$ & & $\mathrm{x}$ \\
\hline Camponotus rufipes (Fabricius, 1775) & & $\mathrm{x}$ & & \\
\hline Cephalotes atratus (Linnaeus, 1758) & $\mathrm{x}$ & & & \\
\hline Cephalotes pusillus (Klug, 1824) & $\mathrm{X}$ & $\mathrm{x}$ & & \\
\hline Crematogaster evallans Forel, 1907 & $\mathrm{x}$ & $\mathrm{x}$ & $\mathrm{X}$ & \\
\hline Crematogaster sp. 1 & $\mathrm{X}$ & $\mathrm{x}$ & & \\
\hline Ectatomma brunneum Smith, 1858 & & $\mathrm{X}$ & & \\
\hline Ectatomma permagnum Forel, 1908 & & & & $\mathrm{x}$ \\
\hline Gnamptogenys moelleri (Forel, 1912) & & $\mathrm{x}$ & & $\mathrm{x}$ \\
\hline Labidus coecus (Latreille, 1802) & & $\mathrm{X}$ & & \\
\hline Labidus praedator (Smith, 1858) & & $\mathrm{x}$ & & \\
\hline Nesomyrmex sp. & $\mathrm{x}$ & & & \\
\hline Linepithema humile (Mayr, 1868) & & $\mathrm{x}$ & & \\
\hline Linepithema sp.2 & & $\mathrm{X}$ & & \\
\hline Mycocepurus sp.1 & & $\mathrm{x}$ & & $\mathrm{X}$ \\
\hline Neoponera vilosa (Fabricius,1804) & & $\mathrm{x}$ & & \\
\hline Odontomachus bauri Emery, 1892 & & $\mathrm{X}$ & & \\
\hline Odontomachus sp.1 & & & $\mathrm{x}$ & $\mathrm{x}$ \\
\hline Pachycondyla striata Smith, 1858 & & & & $\mathrm{x}$ \\
\hline Pheidole sp.2 & $\mathrm{X}$ & $\mathrm{x}$ & & \\
\hline Pheidole sp.6 & $\mathrm{X}$ & & & \\
\hline Pseudomyrmex sp.1 & & $\mathrm{x}$ & & \\
\hline Pseudomyrmex sp.2 & & $\mathrm{x}$ & & \\
\hline Sericomyrmex sp.1 & $\mathrm{X}$ & $\mathrm{X}$ & & \\
\hline Solenopsis invicta Buren, 1972 & $\mathrm{X}$ & & & \\
\hline Solenopsis sp.2 & & $\mathrm{x}$ & & \\
\hline Mycetomoellerius sp.1 & & $\mathrm{X}$ & & \\
\hline Riqueza de espécies & 12 & 21 & 4 & 7 \\
\hline
\end{tabular}




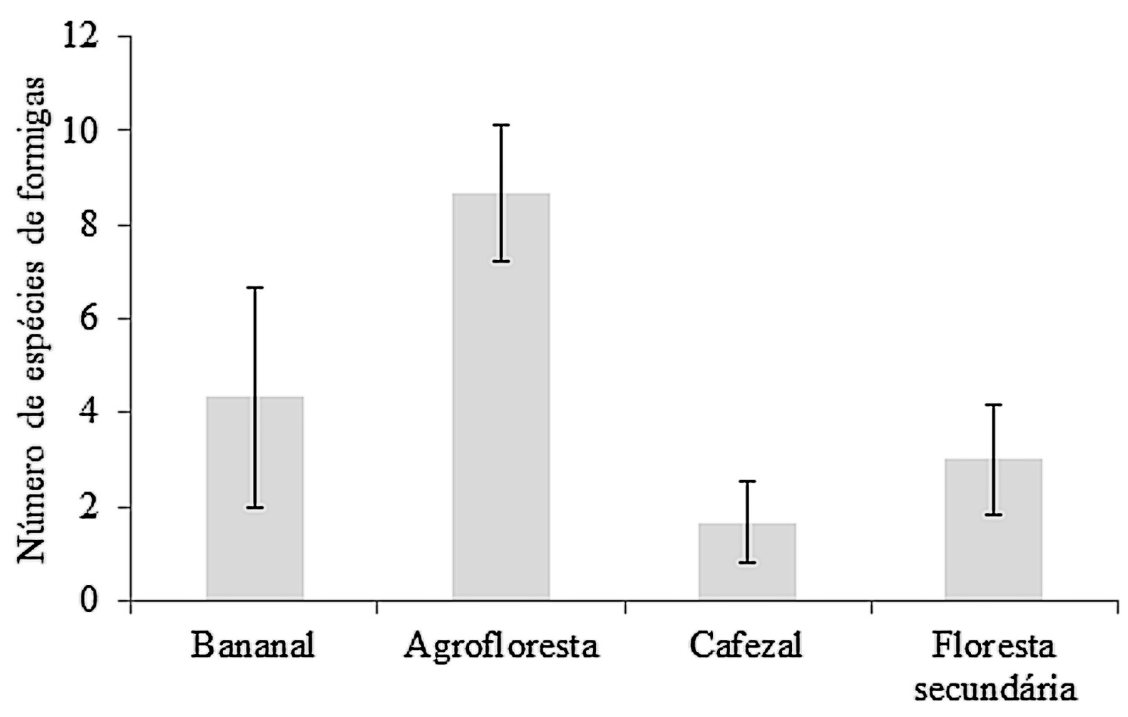

Usos do solo

Figura 1 - Número médio de espécies de formigas ( $\pm \mathrm{EP}$ ) em áreas com diferentes usos do solo no SIPA, Seropédica/RJ.

Tabela 2 - Lista de interações ecológicas observadas no SIPA, Seropédica/RJ.

\begin{tabular}{|l|l|}
\multicolumn{1}{c|}{ Espécies } & \multicolumn{1}{c|}{ Interaçóes ecológicas } \\
\hline Atta sexdens (Linnaeus, 1758) & Herbivoria sobre café \\
\hline Camponotus crassus Mayr, 1862 & Mirmecofilia em pulgão \\
\hline Camponotus novogranadensis Mayr, 1870 & Mirmecofilia em cochonilha \\
\hline Cephalotes astratus (Linneaus, 1758) & Mirmecofilia em cochonilha \\
\hline Cephalotes pusillus (Klug, 1824) & Mirmecofilia em cochonilha \\
\hline Mycocepurus sp.1 & Transportando material vegetal não identificado \\
\hline Pachycondyla striata Smith, 1858 & Predação de lagarta \\
\hline Pheidole sp. 2 & Predação de artrópode não identificável \\
\hline Pheidole sp. 2 & Consumo de estigma de flor \\
\hline Pheidole sp.6 & Predando e carregando anelídeo \\
\hline Pseudomyrmex sp.1 & Mirmecofilia em pulgões \\
\hline Pseudomyrmex sp. 2 & Predação em Pheidole sp.2 \\
\hline
\end{tabular}

Queiroz et al. (2006) e Santo (2007) avaliaram que, durante a atividade de forrageio, as formigas dispersam sementes, algumas predam variadas espécies (Hölldobler \& Wilson, 1990; Baccaro, 2015), utilizam exsudados de plantas e visitam flores (Dátillo et al., 2009; Melo, 2019), as quais são atividades de elevada importância na manutenção do equilîbrio dos ecossistemas.
As formigas do gênero Camponotus são geralmente onívoras e nidificam e forrageiam sobre solo e plantas (Baccaro et al., 2015). Teveram a maior riqueza em todos os ambientes $e$ nos dois estratos amostrais, com o total de quatro indivíduos. No cafezal, um dos ambientes mais homogêneos deste estudo os indivíduos da espécie Camponotus crassus Mayr, 1862 foram observados 
interagindo com pulgões; e na agroflorestal, as operárias de Camponotus novogranadensis Mayr, 1870 interagiam com cochonilhas. Ambas as espécies são fitófagas, cochonilhas e pulgões, e prejudicam o desenvolvimento fitofisiológico, causando a perda da produtividade da cultura. As formigas desse gênero podem se associar a hemípteros fitófagos, que são pragas agrícolas, favorecendo o crescimento populacional dos insetos sugadores (Fleig, 2011), como também atuam como predadores de outros insetos (Santos \& Resende, 1996), podendo ser úteis contra herbívoros (Oliveira et al., 1987; Oliveira \& Brandão 1991). Damon \& Perez-Soriano (2005), ao observarem o comportamento das operárias de C. novogranadensis estas defendem plantas com nectários extraflorais, outra situação é que sua alimentação provém de fezes açucaradas de hemípteros sugadores (Letourneau \& Choe, 1987).

Relacionando as espécies Camponotus crassus Mayr, 1862 e Camponotus rufipes (Fabricius, 1775) como exemplo, elas são frequentes em áreas cultivadas (Almeida et al., 2007) e, quando presentes na espécie Caryocar brasiliense (Pequi), evitam a oviposição por borboletas (Sendoya et al., 2009).

A espécie Atta sexdens (Linnaeus, 1758) (saúva-limão) é uma das principais pragas agrícolas no Brasil por ser desfolhadora de várias culturas (Della Lucia, 2003). Foi localizada no plantio de café, onde realizaram a retirada dos grãos em processo de decomposição dos ramos e grãos caídos ao solo. Além dessa interação negativa para as plantas, as saúvas cultivam fungos para a sua alimentação e já foram observadas em competição com outras espécies de formigas.

Fujihara et al. (2013) mencionam que as formigas do gênero Atta necessitam de elevada quantidade de material vegetal, utilizando plantas em todas as fases de desenvolvimento, e apresentam vasta distribuição geográfica. Essa espécie provavelmente não foi observada na agrofloresta devido à sua preferência por locais abertos (Zanetti et al., 2014). Formigas dos gêneros Mycocepurus e Mycetomoellerius (ver Trachymyrmex, Solomon et al., 2019) também são cultivadoras de fungos e, geralmente, não são consideradas pragas agrícolas, embora existam relatos de formigas desses gêneros acarretando danos às plantas (Insberger, 2007).
Cephalotes é um gênero de formigas onívoras tipicamente arborícolas, mas que também podem ser observadas forrageando sobre o solo, como foi observado no presente estudo (Baccaro et al., 2015). De acordo com Sendoya (2009) e Byk \& Del-Claro (2010), as espécies deste gênero não contribuem para a proteção de plantas, não atacam agentes herbívoros, e reduzirem a fertilização pelo consumo de pólen. Além disso, operárias de Cephalotes pusillus (Klug,1824) (DelClaro et al., 2002) foram observadas interagindo com cochonilhas na agrofloresta, portanto, não sendo uma interação positiva para os cultivos. No entanto, Madureira e Sobrinho (2002) observaram que as formigas do gênero Cephalotes sp. se relacionavam mutualisticamente com Qualea cordata Spreng., protegendo a planta contra hebivoria do gênero Atta.

Formigas do gênero Crematogaster também forrageiam frequentemente sobre plantas (Feldharr et al., 2016) e são onívoras-detritívoras, incluindo a espécie Crematogaster evallans Forel, 1907 (Silvestre et al., 2003). Esse gênero foi observado no bananal, na agrofloresta e no cafezal, forrageando no solo e sobre as plantas, não demonstrando uma ação nociva ao plantio.

O gênero Pheidole, o mais diverso dentre os gêneros de Formicidae, apresenta 1003 espécies conhecidas pela ciência, e essa elevada diversidade taxonômica no mundo acarreta em grande diversidade local, pois geralmente é o gênero com maior riqueza de espécies em levantamentos da fauna de formigas brasileira (Vicente et al., 2018; Jory \& Feitosa, 2020). Entretanto, apesar de apresentar um comportamento onívoro, esse gênero foi apenas observado no bananal $e$ na agrofloresta. Esse gênero, juntamente com Crematogaster e Solenopsis, está entre os mais comuns predadores de pragas do algodoeiro (Bastos et al., 2005). Na plantação de bananeiras, indivíduos do Pheidole foram observados predando artrópodes e um anelídeo, o qual não demostra nocividade ao local, mas sua predação causada a este indivíduo possui um caráter nocivo ao desenvolvimento do ambiente.

O gênero Solenopsis apresenta formigas com hábitos alimentares e de nidificação variados, embora forrageiem e formem seus ninhos principalmente na interface solo-serapilheira (Almeida et al., 2007; Gomes et al., 2013). São recrutadoras eficazes e predadoras vorazes, principalmente de outros insetos. Muitos ninhos da 
espécie Solenopsis invicta Buren, 1972 (formiga lava-pés) são encontrados no SIPA (Almeida et al., 2007) e sabe-se que é predadora de várias espécies de insetos praga em áreas agrícolas (Eubanks, 2001). No entanto, apesar de ter sido observada, não foi registrada nenhuma interação das espécies desse gênero.

O gênero Labidus, cujos indivíduos são chamados vulgarmente de formigas-de-correição, é voraz predador e forrageador subterrâneo, mas pratica ações massivas na superfície da serapilheira e no solo (Bolton, 2015; Brandão, Silva \& Delabie, 2012). É um grupo funcional de predadoras em massa que estavam presentes no sistema agroflorestal neste estudo, as quais são encontradas frequentemente em florestas primárias (García-Martínez et al., 2015). Existem poucas informações sobre as suas atividades e o quanto elas afetam a superfície do solo na predação de artrópodes (Berghoff et al., 2003).

As espécies Pachycondyla striata Smith, 1858, Neoponera vilosa (Fabricius, 1804) e Odontomachus bauri Emery, 1892, pertencentes à subfamília Ponerinae, são caracterizadas como predadoras que se alimentam principalmente de outros artrópodes; contudo, podem consumir partes nutritivas de sementes e frutos, inclusive dispersam sementes na Mata Atlântica (Gianotti \& Machado 2012; Almeida et al., 2013; Amaral et al., 2017).

Essas formigas desempenham um papel importante como bioindicadoras e estão comumente presentes em florestas tropicais; sendo do grupo funcional de formigas predadoras generalistas, se alimentam de uma ampla gama de recursos presentes no ambiente e são predadoras que regulam populações de outros artrópodes (Baccaro et al., 2015). Indivíduos de Pachycondyla striata foram observados na floresta secundária predando e carregando lagarta.

As espécies do gênero Pseudomyrmex, encontradas no presente estudo durante a observação de campo. São arborícolas, nidificando $e$ forrageando sobre plantas em sua grande maioria, inclusive podem proteger as plantas de herbivoria e se alimentam de substâncias secretadas por nectários extraflorais (Heil et al., 2014). Gonzalez-Teuber \& Heil (2010) apresentaram que o mutualismo de formigas da espécie Pseudomyrmex ferruginea Smith, 1877 com acácia combate ameaças microbianas.

\section{Conclusão}

Deste modo, com base nos resultados do presente estudo, podemos constatar um padrão observado em estudos anteriores, de que a fauna de formigas participa de diversas interações ecológicas úteis no desenvolvimento de atividades agrícolas, como predação de outros artrópodes, em diferentes sistemas de plantio e proteção contra a ação de herbívoros. Dentre as áreas cultivadas estudadas, o sistema agroflorestal apresentou o maior potencial para a proteção da fauna de formigas e suas interações ecológicas.

\section{Referências}

Almeida FS \& Vargas AB. Bases para a gestão da biodiversidade e o papel do Gestor Ambiental. Diversidade e Gestão, 1: 10-32, 2007.

Almeida FS, Queiroz JM \& Mayhe-Nunes AJ. Distribuição e abundância de ninhos de Solenopsis invicta Buren (Hymenoptera: Formicidae) em um agroecossistema diversificado sob manejo orgânico. Floresta e Ambiente, 14: 34-44, 2007.

Almeida FS, Mayhe-Nunes AJ \& Queiroz JM. The Importance of Poneromorph Ants for Seed Dispersal in Altered Environments. Sociobiology, 60: 229-235, 2013.

Amaral GCD, Vargas AB \& Almeida FS. Forrageio diurno de Odontomachus bauri Emery (Hymenoptera: Formicidae) em plantio de Eucalyptus sp. no município de Volta Redonda-RJ. EntomoBrasilis, 10(3): 143-147, 2017.

Amaral GCD, Vargas AB \& Almeida FS. Efeitos de atributos ambientais na biodiversidade de formigas sob diferentes usos do solo. Ciência Florestal, 29(2): 660-672, 2019.

Assis DS, Dos Santos IA, Ramos FN, Barrios-Rojas KE, Majer JD \& Vilela EF. Agricultural matrices affect ground ant assemblage composition inside Forest fragments. PloS one, 13(5): e0197697, 2018. https:// doi.org/10.1371/journal.pone.0197697

Baccaro FB et al. 2015. Guia para os gêneros das formigas do Brasil. Editora Inpa: Manaus, 388p.

Bastos CS \& Torres JB. 2005. Controle biológico e o manejo de pragas do algodoeiro. Embrapa AlgodãoCircular Técnica (INFOTECA-E). 63p. <https://www. infoteca.cnptia.embrapa.br/bitstream/doc/278333/1/ CIRTEC72.pdf > Acesso em: 20/04/2018. 
Berghoff SM, Maschwitz U \& Linsenmair KE. Influence of the hypogaeic army ant Dorylus (Dichthadia) laevigatus on tropical arthropod communities. Oecologia, 135(1): 149-157, 2003.

Byk J \& Del-Claro K. Nectar-and pollen-gathering Cephalotes ants provide no protection against herbivory: a new manipulative experiment to test ant protective capabilities. Acta Ethologica, 13(1): 33-38, 2010.

Cantarelli EB, Costa EC, Pezzutti R \& da Silva Oliveira L. Quantificação das perdas no desenvolvimento de Pinus taeda após o ataque de formigas cortadeiras. Ciência Florestal, 18(1): 39-45, 2008.

Damon A \& Pérez-Soriano MA. Interaction between ants and orchids in the Soconusco region, Chiapas, Mexico. Entomotropica, 20(1): 59-65, 2005.

Dáttilo W, da Costa Marques E, de Faria Falcão JC \& Oliveira Moreira DD. Interações mutualísticas entre formigas e plantas. EntomoBrasilis, 2(2): 32-36, 2009.

Della Lucia MTC. 2003. Hormigas de Importancia Económica em la Región Neotropical. In: Fernandez $\mathrm{F}$, (ed.). Introductión a las hormigas de la región Neotropical. Acta Noturna, Bogotá, Colombia. 337p.

Del-Claro K, Santos JC \& Júnior ADS. Etograma da formiga arborícola Cephalotes pusillus (Klug, 1824) (Formicidae: Myrmicinae). Revista de Etologia, 4(1): 31-40, 2002.

Estrada ME. 2017. A diversidade e o papel da fauna de formigas em áreas agrícolas submetidas ao cultivo orgânico e convencional. Dissertação (Mestrado em Fitossanidade e Biotecnologia Aplicada). Universidade Federal Rural do Rio de Janeiro. 90p.

Eubanks MD. Estimates of the direct and indirect effects of red imported fire ants on biological control in field crops. Biological Control, 21: 35-43, 2001.

Feldhaar H, Maschwitz U \& Fiala B. Taxonomic Revision of the obligate plant-ants of the genus Crematogaster Lund (Hymenoptera, Formicidae, Myrmicinae), associated with Macaranga Thouars (Euphorbiaceae) on Borneo and the Malay Peninsula. Sociobiology, 63(1): 651-681, 2016.

Ferreira AC. 2016. O Gênero Pheidole (Hymenoptera: Formicidae: Myrmicinae) no Paraná: Levantamento e delimitação de espécies. Tese de Mestrado. PósGraduação em Ciências Biológicas. Universidade Federal do Paraná. 387p.

Fillman DA \& Sterling WL. Killing power of the red imported fire ant [Hym.:Formicidae]: a key predator of the boll weevil [Col.: Curculionidae]. Entomophaga, 28(4): 339-344, 1983.
Fleig ED. 2011. Estudo da interação entre a formiga Camponotus punctulatus Mayr (Hymenoptera: Formicidae) e o pulgão-preto-dos-citros Toxopteraci tricidus (Kirkaldy) (Hemiptera: Aphididae). Tese (Doutorado). Universidade Federal do Rio Grande do Sul, Programa de Pós-Graduação em Fitotecnia. $109 \mathrm{p}$.

Forti LC. Se o produtor vacilar, o exército das formigas invade a lavoura. Revista Granja, 56: 12-17, 2000.

Fujihara RT, de Souza Silva M \& Forti LC. 2013. Manejo de Formigas Cortadeiras. In: Baldin ELL, Fujihara RT \& Souza A. Tópicos Especiais em Proteção de Plantas. Fundação de Estudos e Pesquisas Agrícolas e Florestais. Fundação de Estudos e Pesquisas Agrícolas e Florestais. 1a ed. Botucatu-SP.164p.

Giannotti E \& Machado VLL. Notes on the foraging of two species of Ponerine ants: food resources and daily hunting activities (Hymenoptera: Formicidae). Bioikos, 6: 7-17, 1992.

Gomes DS, Almeida FS, Vargas AB \& Queiroz JM. Resposta da assembleia de formigas na interface soloserapilheira a um gradiente de alteração ambiental. Iheringia. Série Zoologia, 103: 104-109, 2013.

González-Teuber M \& Heil M. Pseudomyrmex ants and Acacia host plants join efforts to protect their mutualism from microbial threats. Plant signaling \& behavior, 5(7): 890-892, 2010.

Gravena S. Cochonilha-branca: descontrolada em 2001. Laranja, Cordeirópolis, 24: 71-82, 2003.

Hammer O, Harper DAT \& Ryan PD. Paleontological Statistics - PAST. 2003. Disponível em: < http://folk.uio. no/ohammer/past>. Acesso em: 08 janeiro 2017.

Heil M, Barajas-Barron, A, Orona-Tamayo, D, Wielsch $\mathrm{N}$ \& Svatos A. Partner manipulation stabilises a horizontally transmitted mutualism. Ecology letters, 17(2): 185-192. 2014.

Hölldobler B \& Wilson EO. 1990. The ants. Harvard University Press. 732p.

Insberger TK. 2007. Identificação das formigas cortadeiras coletadas em área para o cultivo de Pinus. Trabalho de Conclusão de Curso (Bacharel em Ciências Biológicas), Universidade do Contestado. 49p.

Jory TT \& Feitosa RM. First survey of the ants (Hymenoptera, Formicidae) of Piauí: filling a major knowledge gap about ant diversity in Brazil. Papéis Avulsos de Zoologia, 60: e20206014, 2020. 
Letourneau DK \& Choe JC. Homopteran attendance by wasps and ants: the stochastic nature of interactions. Psyche, 94(1-2): 81-89, 1987.

Madureira M \& Sobrinho TG. Evidência de mutualismo entre Qualea cordata (Vochysiaceae) e Cephalotes sp.(Hymenoptera: Formicidae). Academia Insecta, 2(1): 1-4, 2002.

Martins L, Almeida FS, Mayhe-Nunes AJ \& Vargas AB. Efeito da complexidade estrutural do ambiente sobre as comunidades de formigas (Hymenoptera: Formicidae) no município de Resende, RJ, Brasil. Revista Brasileira de Biociências (Online), 9: 174-179, 2011.

Melo HCL. 2019. Interação planta-formiga: modulação da produção de néctar extrafloral e resposta defensiva por formiga. Monografia (Graduação em Ciências Biológicas) - Departamento de Biologia, Centro de Ciências Biológicas e da Saúde, Universidade Federal de Sergipe, São Cristóvão. 23p.

Neves MCP, Guerra JGM, Carvalho SR, Ribeiro RLD \& Almeida DL. 2005. Sistema integrado de produção agroecológica ou fazendinha agroecológica do km 47. p. 147-172. In: Aquino A \& Assis RL (Org.). Agroecologia: princípios e técnica para uma agricultura orgânica sustentável. Brasília: Embrapa-Informação Tecnológica. 517p.

Nóbrega PDO. 2006. Implantação, manejo e aporte de nutrientes em agrofloresta em um sistema orgânico de produção. Monografia (Graduação em Engenharia Florestal) - Instituto de Florestas, Universidade Federal Rural do Rio de Janeiro, Seropédica, 48p.

Oliveira PS. 1991.The ant community associated with extrafloral nectaries in the Brazilian cerrados. Ant-plant interactions.

Oliveira OS, da Silva AF \& Martins AB. Ant foraging on extrafloral nectaries of Qualea grandiflora (Vochysiaceae) in cerrado vegetation: ants as potential antiherbivore agents. Oecologia, 74(2): 228-230, 1987.

Passos L \& Oliveira PS. Ants affect the distribution and performance of Clusiacriuva seedlings, a primarily bird-dispersed rain forest tree. Journal of Ecology, 90: 517-528, 2002.

Peres MB, Vercillo UE \& de Souza Dias BF. Avaliação do Estado de Conservação da Fauna Brasileira e a Lista de Espécies Ameaçadas: o que significa, qual sua importância, como fazer? Biodiversidade Brasileira, 1(1): 45-48, 2011.

Pimm SL et al. The biodiversity of species and their rates of extinction, distribution, and protection. Science, 344(6187): 1-12, 2014.
Rodrigues CA, Da Silva Araújo M, Cabral PID, Lima R, Bacci L \& Oliveira MA. Comunidade de formigas arborícolas associadas ao pequizeiro (Caryocar brasiliense) em fragmento de Cerrado Goiano. Pesquisa Florestal Brasileira, 57: 39-44, 2008.

Rodrigues DM, Ferreira LO, da Silva NR, Santos Guimarães ED, Martins ICF \& Assis Oliveira FD. Diversidade de artrópodes da fauna edáfica em agroecossistemas de estabelecimento agrícola familiar na Amazônia Oriental. Revista de Ciências Agrárias/ Amazonian Journal of Agriculturaland Environmental Sciences, 59(1): 32-38, 2016.

Santo MME. Secondary seed dispersal of Ricinus communis Linnaeus (Euphorbiaceae) by ants in secondary growth vegetation in Minas Gerais. Revista Árvore, 31: 1013-1018, 2007.

Santos GM de M \& Resende JJ. Predação de Syntermes molestus (Burmeister, 1839) (Isoptera-Termitidae) por Camponotus blandus (Fr. Smith, 1858) (HymenopteraFormicidae) em Feira de Santana/Ba. Sitientibus, 15: 175-182, 1996.

Sendoya SF, Freitas AVL \& Oliveira PS. Egg-laying butterflies distinguish predaceous ants by sight. Amernat, 174: 134-140, 2009.

Silva ERDA. 2012. Efeito de borda sobre a comunidade de formigas em remanescentes de mata atlântica nordestina em relação ao agroecossistema de canade-açúcar. Tese de Mestrado em Ciências Florestais. Universidade Federal Rural de Pernambuco, 70p.

Solomon SE et al. The molecular phylogenetics of Trachymyrmex Forel ants and their fungal cultivars provide insights into the origin and coevolutionary history of 'higher-attine' ant agriculture. systematic Entomology, 44(4): 939-956, 2019.

Tabarelli $\mathrm{M}$ et al. A conversão da floresta atlântica em paisagens antrópicas: lições para a conservação da diversidade biológica das florestas tropicais. Interciencia, 37(2): 88-92, 2012.

Rodrigues CA, da Silva Araújo M, Cabral PID, Lima $\mathrm{R}$, Bacci L \& Oliveira MA. Comunidade de formigas arborícolas associadas ao pequizeiro (Caryocar brasiliense) em fragmento de Cerrado Goiano. Pesquisa Florestal Brasileira, 57: 39-44, 2008.

Vicente RE, Ferreira AC, Santos RCL \& Prado LP. Ants (Hymenoptera: Formicidae) from an Amazonian fragmented landscape, Juara, Mato Grosso, Brazil, with new records of ant species. Papéis Avulsos de Zoologia, v.58, e20185840. Epub, 2018. 
Wilson EO. The arboreal ant fauna of Peruvian Amazon forests: a first assessment. Biotropica, 245-251, 1987.
Zanetti R, Zanuncio JC, Santos JC, Silva WLP, Ribeiro GT \& Lemes PG. An overview of integrated management of leaf-cutting ants (Hymenoptera: Formicidae) in brazilian forest plantations. Forests, 5: 439-454, 2014.

Biodiversidade Brasileira - BioBrasil.

Fluxo Contínuo

n. 1,2021

http://www.icmbio.gov.br/revistaeletronica/index.php/BioBR

Biodiversidade Brasileira é uma publicação eletrônica científica do Instituto Chico Mendes de

Conservação da Biodiversidade (ICMBio) que tem como objetivo fomentar a discussão e a disseminação de experiências em conservação e manejo, com foco em unidades de conservação $e$ espécies ameaçadas.

ISSN: $2236-2886$ 